\title{
Interaction between a laser beam and semiconductor nanowires: application to the Raman spectrum of $\mathrm{Si}$ nanowires
}

\author{
J. Anaya , A. Torres, A.C. Prieto and \\ J. Jimenez
}

\section{A. Rodríguez and T. Rodríguez}

\begin{abstract}
One presents in this work the study of the interaction between a focused laser beam and Si nanowires (NWs). The NWs heating induced by the laser beam is studied by solving the heat transfer equation by finite element methods (FEM). This analysis permits to establish the temperature distribution inside the NW when it is excited by the laser beam. The overheating is dependent on the dimensions of the NW, both the diameter and the length. When performing optical characterisation of NWs using focused laser beams, one has to consider the temperature increase introduced by the laser beam. An important issue concerns the fact that the NW's diameter has subwavelength dimensions, and is also smaller than the focused laser beam. The analysis of the thermal behaviour of the NWs under the excitation with the laser beam permits the interpretation of the Raman spectrum of Si NWs. It is demonstrated that the temperature increase induced by the laser beam plays a major role in shaping the Raman spectrum of Si NWs.
\end{abstract}

Keywords: Si; nanowires; Raman spectroscopy; thermal conductivity; laser heating; phonons. 
Biographical notes: $\mathrm{J}$. Anaya is a $\mathrm{PhD}$ student at the Optronlab research group in the University of Valladolid. Spain. His current research focuses on semiconductor nanostructures based on nanowires.

A. Torres is currently an Associated Researcher at the Mechanical Engineering School, University of Valladolid, Spain. He received his $\mathrm{PhD}$ from the University of Valladolid. His current research interests are concerned on semiconductor nanostructures both nanowires and thin films.

A.C. Prieto is a Professor at the University of Valladolid, Spain. He received his $\mathrm{PhD}$ from the University of Valladolid (Spain). His current research interests include the characterisation by Raman spectroscopy of nanostructural material semiconductors

J. Jiménez is a Full Professor of Condensed Matter Physics at the University of Valladolid. He received his $\mathrm{PhD}$ from the Universities of Valladolid and Montepellier (France). His current research activity includes optical characterisation of semiconductors and nanowires, using cathodoluminescence, photoluminescence and Raman spectroscopy techniques.

A. Rodríguez is an Associate Professor at the Telecommunication Engineering School, Technical University of Madrid, Spain. He received his $\mathrm{PhD}$ in Electronic Engineering from the same university. His current research interests include nanostructured materials and thin films

T. Rodríguez is a Full Professor at the E.T.S.I Telcomunicación in the Polytechnic University of Madrid (Spain). His current research interest is Group IV semiconductor heterostructures based on nanowires.

This paper is a revised and expanded version of a paper entitled 'Interaction between a laser beam and semiconductor nanowires: application to the Raman spectrum of $\mathrm{Si}$ nanowires' presented at the 11th International Workshop on Beam Injection Assessment of Microstructures Annaba, Algeria, 25-28 June 2012.

\section{Introduction}

Semiconductor nanowires (NWs) have a strong potential in electronics and optoelectronics; e.g., field effect transistors, interconnects, heterostructured devices, thermoelectric converters, photovoltaics, and sensors (Cui and Lieber, 2001; Kayes et al., 2005; Hochbaum et al., 2008). Besides the quantum confinement effects, relevant for NWs with diameter below -20 nm (Campbell and Fauchet, 1986; Piscanec et al., 2003), the high aspect ratio of NWs with diameters sensibly larger than the limit for quantum effects also present noteworthy changes of the physical properties as compared to the corresponding bulk semiconductors. In particular, the thermal conductivity of NWs is strongly reduced with respect to their bulk counterparts ( $\mathrm{Li}$ et al., 2003a). This is a very important issue, because the thermal transport is critical for the devices based on NWs. The size reduction leads to an increase in the power density to be dissipated in spite of the lower current operation; therefore, the heat extraction from the active zones of the devices is crucial for achieving high performance and reliable nanodevices. 
Characterisation techniques are necessary in order to understand the physical properties of semiconductor NWs, which are limited not only by the dimension, but also, by other features as the surface roughness. Optical characterisation tools with submicrometer spatial resolution are necessary to boost the understanding of these structures in view of the development of new nanodevice concepts. Lasers focused down to submicrometric probe beams are non invasive excitation sources allowing for the study of the fundamental properties of semiconductors. In particular, photoluminescence (PL) (Liu et al., 2010), optical absorption (OA) (Xie et al., 2011), Raman scattering (RS) (Piscanec et al., 2005; Adu et al., 2006; Doerk et al., 2009; Torres et al., 2010; Soini et al., 2010; Alarcón-Lladó et al., 2011), and photocurrent (PC) (Ahn et al., 2005), are characterisation tools for diverse semiconductor nanostructures. NWs are one dimensional structures with diameter below the size of the focused laser beam; besides, the laser wavelength is also larger than the NW diameter. Therefore, the interaction between the laser beam and the NWs does not follow the same trends as described for bulk semiconductors. In order to interpret the spectroscopic data, one needs of the understanding of the interaction between the NWs and the laser beam. Another relevant point concerning the dimensions of the NWs, is the small amount of matter probed by the laser beam; which is detrimental to the signal detection. RS is a second order optical process, for which the efficiency is very low, as compared to first order processes, as OA and PL. Usually, the Raman signal of a single NW is insufficient; therefore, one performs the Raman spectrum on ensembles of NWs. When several NWs are studied the optical response arises from a non-homogeneous medium, composed by NWs that can have different dimensions, and are also excited under different conditions as we will discuss later on. On the other hand, recording the Raman spectrum of an individual NW is very challenging, because of the very small volume of scattering.

The semiconductor NWs are systems with poor thermal conductivity, therefore, they are heated by the laser beam, which hinders the interpretation of the experimental results as reported in the literature (Piscanec et al., 2005; Adu et al., 2006; Doerk et al., 2009; Torres et al., 2010; Soini et al., 2010; Alarcón-Lladó et al., 2011). Therefore, an exhaustive study of the interaction of the NWs with the laser beam, taking account of the energy transferred to the NW, is necessary to interpret the spectral data in experiments in which NWs are excited by focused laser beams. Furthermore, the optical measurements can be used as contact less methods for the measurement of the thermal conductivity, for which one needs to have a full description of the interaction between the laser beam and the NW.

We present herein the analysis of the interaction between the laser beam and Si NWs, and the application to the interpretation of the Raman spectra of NWs.

\section{Absorption efficiency of NWs}

The measurement of the optical response of NWs is conditioned by the fact that the exciting laser beam diameter at the focus largely exceeds the NW diameter. To get a reasonably signal, one measures ensembles of NWs, which often present diameter and length distributions. On the other hand, NWs have poor thermal conductivity compared to the bulk semiconductors; besides, the poor thermal contact to the substrate suppresses the heat dissipation and the NWs can reach temperatures well above room temperature, to the extreme that this NW heating by the laser beam can provide the main contribution to the 
shape of the optical response of the NWs, e.g., the Raman phonon bands are broadened and downshifted as a consequence of the laser induced heating. Besides, one has to consider that the laser beam presents a Gaussian power profile, the diameter of the laser beam being larger than the NWs diameter $(\approx 1\lceil\mathrm{~m}$ FWHM); therefore, the energy transferred to the NW by the laser beam must depend on the position of the NW inside the laser beam spot; which means that when exciting with the laser beam a bundle of NWs, all of them are not under the same excitation conditions.

On the other hand, because of the sub-wavelength dimension of the NW diameter, and the dielectric mismatch between the NW and the surrounding media, absorption resonances for NW diameters commensurate with the wavelength occur (Doerk et al., 2010). It is possible to calculating the absorption efficiency of NWs, defined in the frame of the Mie theory, as a dimensionless magnitude corresponding to the ratio between the absorption cross section and the projected area of the NW, using Mie solutions of the Maxwell equations (Kerker, 1969), allowing us to handle the true energy absorbed by the Si NW, depending on its diameter and laser wavelength. The absorption efficiency calculated for two different laser wavelengths, showing resonances for certain diameters are shown in Figure 1.

Figure 1 Absorption efficiency for two laser lines $(514.5 \mathrm{~nm}$ and $632 \mathrm{~nm})$ as function of the $\mathrm{Si}$ NW diameter (see online version for colours)

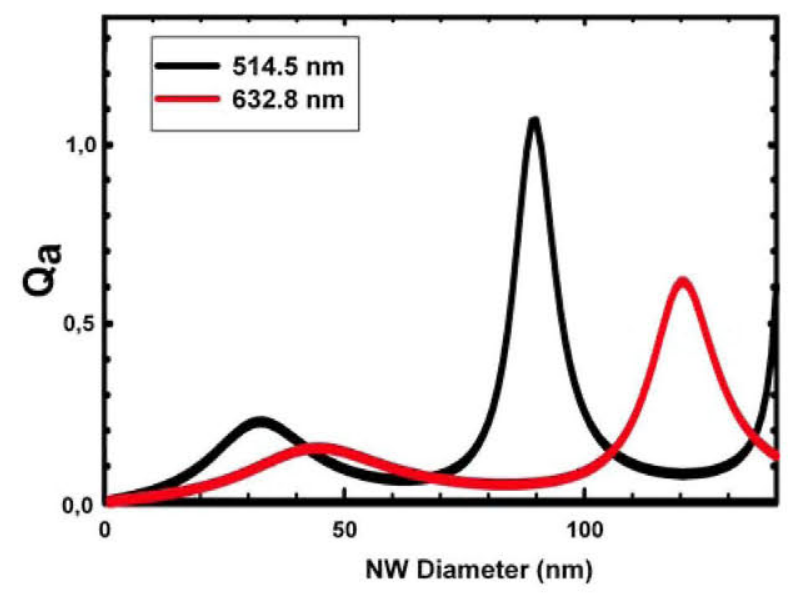

\section{Thermal transport in the NW under the laser beam excitation}

The laser beam interacting with the NW acts as a local heat source. The heat generated at the zone of laser impact is distributed over the NW, which the overheating depends on the effective power absorbed, and the thermal conductivity of the NW (Li et al., 2003a, 2003b), which both of them depending on the NW diameter, and the heat dissipation, which is determined by the thermal contact between the NW and the supporting substrate, and the immersion medium; therefore, one should consider the dimension of the NWs and the surrounding medium. All these aspects need to be considered when solving the heat transport equation inside the $\mathrm{NW}$, in order to give an estimation of the temperature inside the NW excited by the laser beam. 
The heat transfer equation is solved by finite element methods (FEM), using a cylindrical geometry with a mesh composed of more than 500,000 tetrahedra in order to achieve a precise calculation. The heat source is constituted by the laser energy locally absorbed by the NW. One assumes steady state conditions, with natural convective/radiative heat exchange between the NWs and the immersion medium, Neumann boundary conditions for free standing NWs, and Dirichlet boundary conditions in the case of NWs with heatsinks, which corresponds to a fixed temperature of $300 \mathrm{~K}$ in the heatsink, no matter the nature of the heatsink, which can be a Si substrate or a metal pad. The heat distribution in the NW was calculated as a function of the NW dimensions, both length and diameter. First, we studied the temperature reached by a Si NW of $37 \mathrm{~nm}$ diameter, with a thermal conductivity of $18 \mathrm{~W} / \mathrm{mK}$ at $300 \mathrm{~K}$ instead of the $149 \mathrm{~W} / \mathrm{mK}$ of bulk $\mathrm{Si}$ at the same temperature (Li et al., 2003a), as a function of the incident laser power. The temperature represented in Figure 2 corresponds to the temperature estimated in the zone of impact of the laser beam. One observes that even at very low incident laser powers the temperature is significantly enhanced. This suggests that the micro-Raman spectra of NWs present, even when the laser power is reduced, a non negligible contribution of temperature. It should be noted that the laser beam can hardly reduce down to the limit for negligible heating of the NW, because of the very low Raman signal, which is especially true when measuring individual NWs

Figure 2 Temperature at the laser impact zone in a Si NW with $37 \mathrm{~nm}$ diameter, and $5 \mu \mathrm{m}$ length, for two different laser lines $(514 \mathrm{~nm}, \mathrm{Qa} \approx 0.21$, bulk Si absorption coeff. $\approx$ $1.47 \times 104 \mathrm{~cm}^{-1}$ and $632 \mathrm{~nm}, \mathrm{Qa} \approx 0.11$, bulk Si absorption coeff. $\approx 4.1 \times 103 \mathrm{~cm}^{-1}$ ) (see online version for colours)

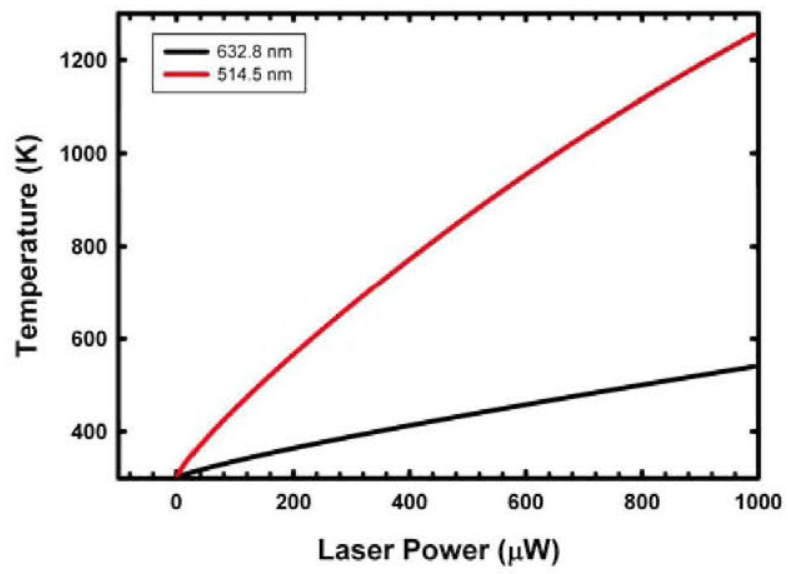

Once the laser impacts on the NW, the energy absorbed constitutes a heat source, being the heat distributed over the NW until reaching the equilibrium between the absorbed and dissipated energy, which depends on the NW dimensions. The temperature at the laser impact zone in a free standing NW is plotted in Figure 3 as a function of the NW diameter.

If one considers the role of the NW length, one observes that the temperature decreases for longer NWs at equal diameter, because of the larger volume of long NWs (not shown here). Furthermore, the temperature distribution inside the NW depends on the position along the NW where the laser beam impacts. 
Figure 3 Temperature (symbols) at the laser impact zone as function of the diameter (fixed length of $5 \mu \mathrm{m}$ ), right axis represents the absorption efficiency (discontinuous line), $\mathrm{QA}$, of the $514.5 \mathrm{~nm}$ laser wavelength

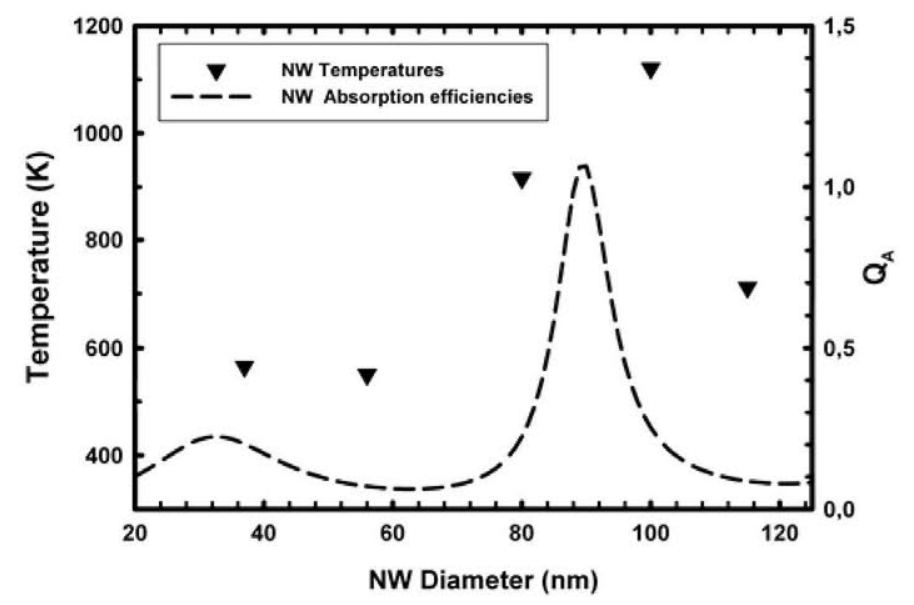

Note: The laser power was settled up at $200 \mu \mathrm{W}$.

Figure 4 Temperature reached by the NW ( $37 \mathrm{~nm}$ diameter, $5 \mu \mathrm{m}$ length) as a function of the position of the NW with respect to the laser beam centre (see online version for colours)

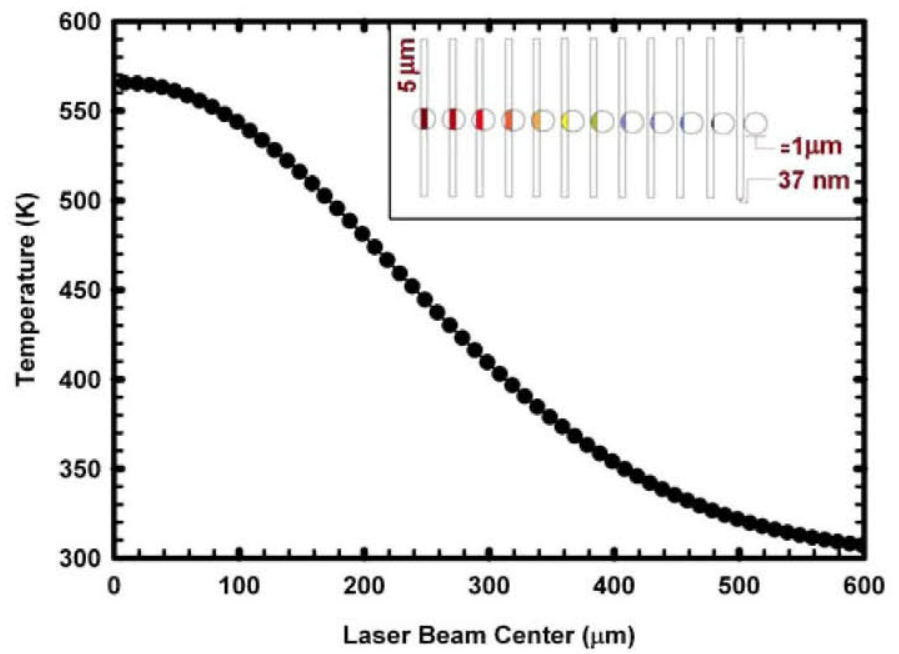

Notes: The origin is the centre of the laser beam spot and the laser moves across the NW in small steps (see inset). The laser used for modelling this configuration was an Ar+ laser at $514.5 \mathrm{~nm}$ and a power of $200 \mu \mathrm{W}$.

A very important aspect concerns the Gaussian power distribution of the laser beam. Because the laser beam spot at focus is larger than the NW diameter, the laser energy absorbed by the NW must strongly depend on the position of the NW inside the focused laser beam spot. This is very important because the NWs diameters are below the diffraction limit, therefore, they can not be resolved in the optical microscope of the micro-Raman apparatus, which makes difficult to achieve a perfect alignment of the NW 
with the laser beam. This is illustrated in the temperature reached by the NW when the laser beam is scanned transversally across the NW in small steps of $0.1 / \mathrm{m}$, Figure 4 . The maximum temperature is reached when the NW is at the centre of the laser beam spot on the focus plane, being notably reduced when the NW is at the periphery of the laser beam spot. This makes sense when measuring ensembles of NWs, because each of the NWs forming the ensemble is excited in a different way in terms of the absorbed energy.

\section{Raman spectrum of NWs}

The Raman spectrum of NWs is sensitive to the diameter of the NWs (Campbell and Fauchet, 1986); the phonon confinement shifts down and asymmetrically broadens the one phonon bands. However, very often shifts of several $\mathrm{cm}^{-1}$ have been reported for the LO-TO phonon band of Si NWs, even with diameters larger than $25 \mathrm{~nm}$, which does not match the expected shift predicted by the Richter, Campbell and Fauchet (RCF) model (Campbell and Fauchet, 1986). According to the discussion of paragraph 3 one can suspect that the Raman spectrum of NWs is shaped by the NW laser induced heating; Stokes /Antistokes intensity ratio confirms NW heating. Several Raman spectra obtained on ensembles of Si NWs are shown in Figure 5. The Raman spectra were acquired with a Labram UV-HR 800 Raman spectrometer from Jobin Yvon. The excitation was done with either a He-Ne laser $(632.8 \mathrm{~nm})$, or an Ar+ laser at $514.5 \mathrm{~nm}$, using a 0.95 numerical aperture 100X objective, thereby obtaining a spot of near $1 \int \mathrm{m}$ in diameter; a laser power on sample surface of $1.3 \mathrm{~mW}$ for He-Neon laser, and and 200 (W for Ar+ laser, were used for spectra acquisition.

Figure 5 Raman spectra of an ensemble of NWs, on the right the scanning electron microscope (SEM) micrograph of the ensemble of NWs (see online version for colours)

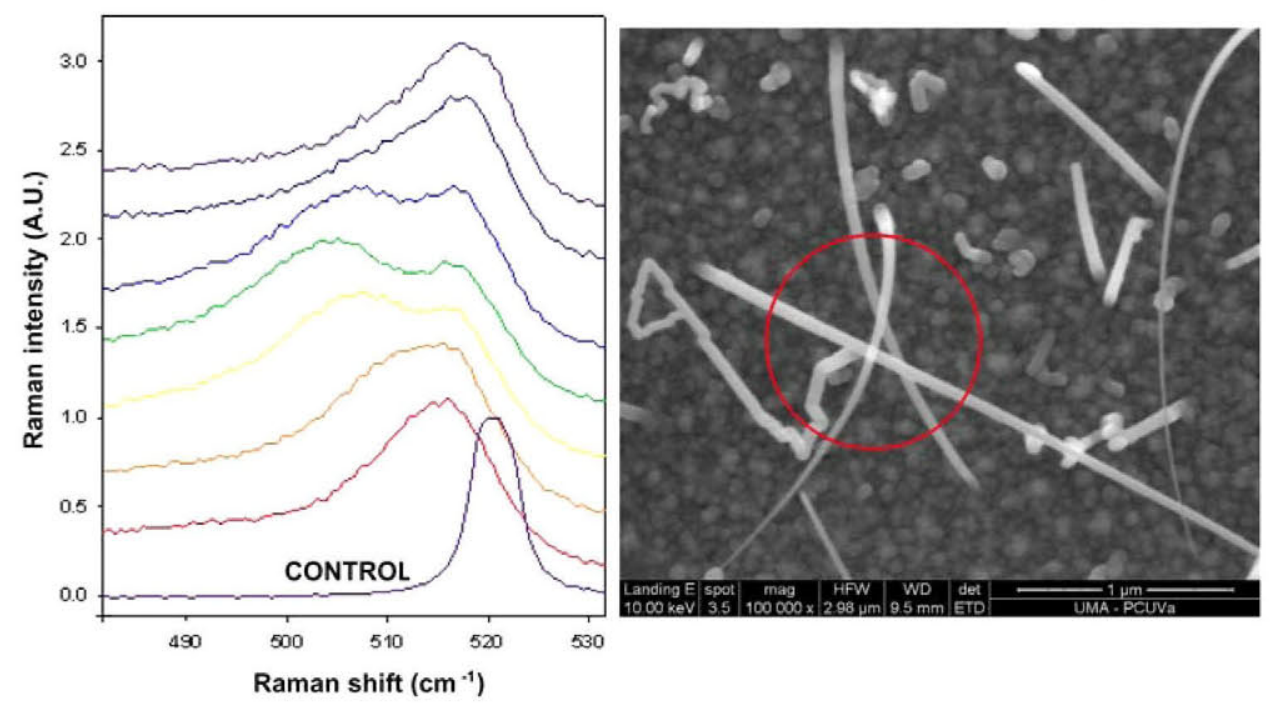

Notes: The control spectrum from a bare $\mathrm{Si}$ is also included. Each spectrum corresponds to a different position of the laser beam. The red circle in the SEM micrograph represents the laser beam spot; one observes that the relative positions of the NWs, taking account of the Gaussian power distribution, must result in dissimilar excitation conditions for each NW, which have also different dimensions. 
One records spectra with several $\mathrm{cm}^{-1}$ shift with respect to the control spectrum of a bare Si substrate. Furthermore, one observes for some of them an asymmetrical broadening, and eventually band splitting. The asymmetry of the Raman bands has been associated with inhomogeneous laser heating (Adu et al., 2006). The Raman signal arises from the volume where the laser impacts, therefore the temperature gradient of interest to the Raman signal is restricted to the laser beam diameter. This very local gradient has been assumed by some authors to be above a few hundred of $\mathrm{K} / / \mathrm{m}$ (Adu et al., 2006); however, there is not evidence that could support this assertion, other than the anomalously large Raman bands observed when measuring ensembles of NWs. We calculated the temperature distribution inside the NW, varying the thermal conductivity down to the lowest thermal conductivity reported for rough NWs, $\kappa \approx 2 \mathrm{~W} / \mathrm{mK}$ (Hochbaum et al., 2008). The temperature gradient at the scale of the laser beam diameter is only a few degrees $/[\mathrm{m}$, even for the NWs with the lowest thermal conductivity; this result rules out the existence of abrupt temperature gradients in a NW at the submicrometric scale. This is confirmed when one measures the Raman spectrum of an individual Si NW, which is heated by the laser beam, but the Raman band appears symmetrical, Figure 6; which rules out the existence of significant temperature gradient inside the scattering volume, in agreement with the calculations.

Figure 6 Raman spectrum of an individual NW (see SEM picture), compared to the control spectrum from a bare $\mathrm{Si}$ substrate (see online version for colours)

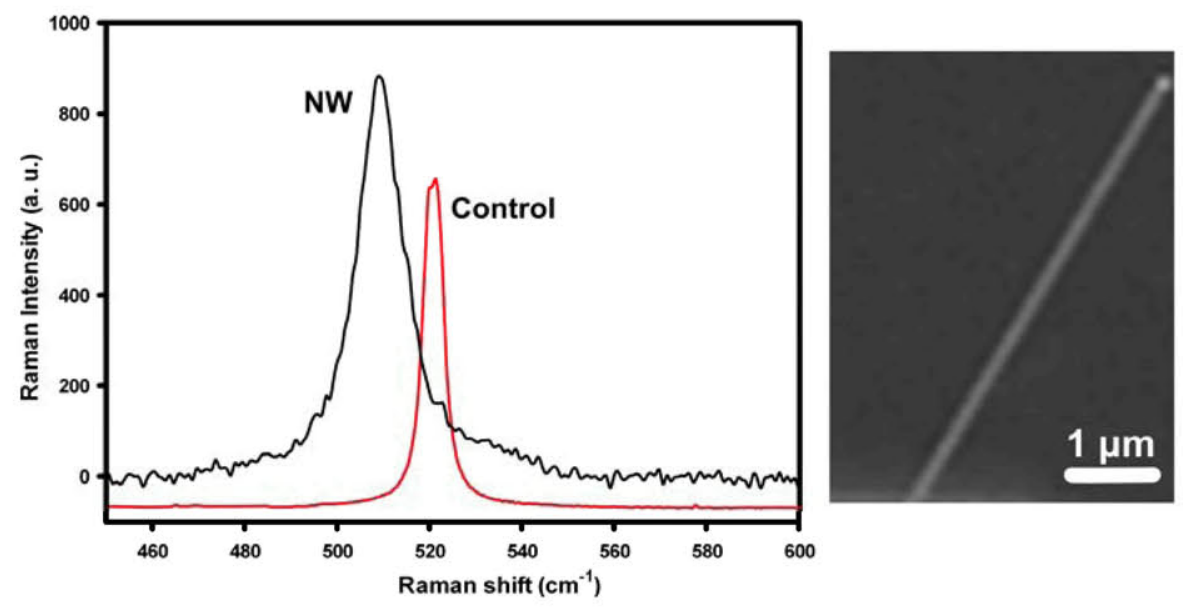

Now the question is: what is the origin of the strong asymmetry observed in the Raman bands of the ensembles of NWs?; as mentioned above the laser beam spot at focus is much larger than the NWs diameters, therefore, several of those NWs are being excited simultaneously, and they are disposed in different positions inside the laser beam spot, which, because of the Gaussian power distribution, are excited at different powers, thereafter they reach different temperatures; therefore, one can argue that depending on the disposition of the NWs inside the laser beam spot the different NWs can coexist at very different temperatures during the Raman measurement. We have simulated the Raman spectrum of a set of four NWs with diameters $25,20,20$ and $20 \mathrm{~nm}$ respectively, lying in the order of magnitude of the diameters observed in the SEM micrograph of 
Figure 5, in two different geometric arrangements inside the laser beam spot of $1 \int \mathrm{m}$ diameter and a laser power of $1.3 \mathrm{~mW}(632.8 \mathrm{~nm})$, Figure 7.

Figure 7 Simulated Raman spectra for each individual NW, and the resultant of the ensemble of four Si NWs (20, 25, 20 and $20 \mathrm{~nm}$ diameter from left to right in the inset), and two different distributions of the NWs inside the laser beam spot (see the insets) (see online version for colours)
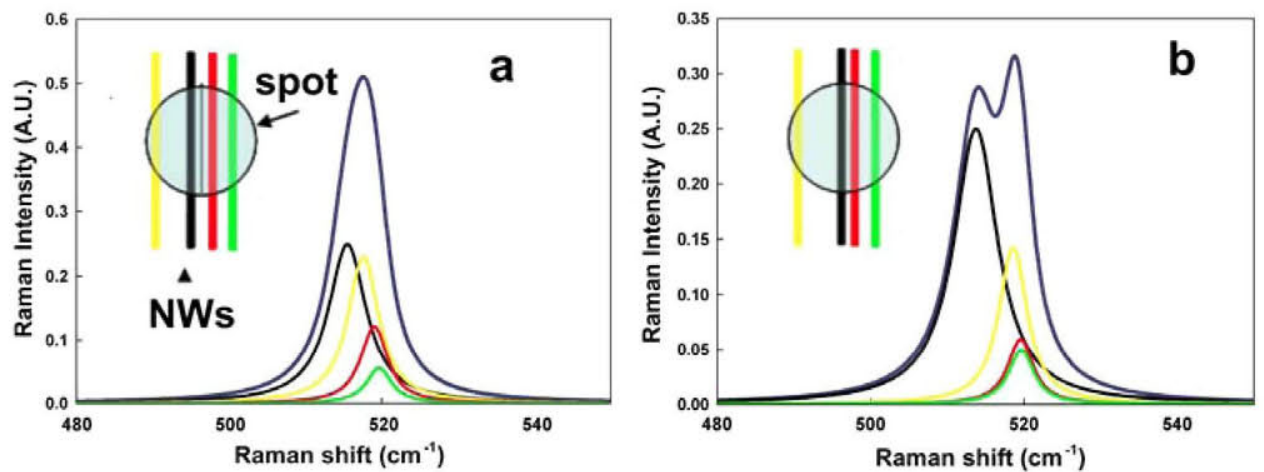

One observes that the resultant spectrum differs from each other configuration. When the temperature reached by the NWs is very different each other, the band splitting, experimentally seen in Figure 5, appears, see Figure 7(b). The anomalously large width of the Raman bands is due to the large temperature in homogeneity, which is consequence of the different temperatures reached by the NWs in different positions with respect to the laser beam axis at the focal plane.

\section{Conclusions}

The temperature induced by the interaction between a focused laser beam and Si NWs has been modelled using FEM methods. The temperature distribution has inside the NWs has been obtained for different excitation conditions; in particular special interest has been paid to the role of the Gaussian power distribution of the laser beam, and the fact that it is larger by at least one order of magnitude than the common NWs diameters. The results obtained permit a full description of the overheating of the NWs excited by the laser beam. This analysis permits to interpret the optical spectroscopy data in NWs excited by focused laser beams. Micro Raman spectra of Si NWs are obtained and interpreted in terms of the heating induced by the laser beam.

\section{Acknowledgements}

This work was funded by the Spanish Government (MAT-2007-66181 and MAT-201020441) and by Junta de Castilla y León (VA051A06-GR202). 


\section{References}

Adu, K.W., Gutiérrez, H.R., Kim, U.J. and Eklund, P.C. (2006) 'Inhomogeneous laser heating and phonon confinement in silicon nanowires: a micro-Raman study', Phys. Rev. B, Vol. 73, No. 15, p. 155333.

Ahn, Y., Dunning, J. and Park, J. (2005) 'Scanning photocurrent imaging and electronic band studies in silicon nanowires field effect transistor', Nanolett, Vol. 5, No. 7, p.1367.

Alarcón-Lladó, E., Ibañez, J., Cuscó, R., Artús, L., Prades, J.D., Estradé, S. and Morante, J.R. (2011) 'Ultraviolet Raman scattering in $\mathrm{ZnO}$ nanowires: quasimode mixing and temperature effects', J. Raman Spectrosc., Vol. 42, No. 2, p.153.

Campbell, I.H. and Fauchet, P.M. (1986) 'The effects of microcrystal size and shape on the one phonon Raman spectra of crystalline semiconductors', Solid St. Commun., Vol. 58, No. 10, p.739.

Cui, Y. and Lieber, C.M. (2001) 'Functional nanoscale electronic devices assembled using silicon nanowire building blocks', Science, Vol. 291, No. 5505, p.851.

Doerk, G.S., Carraro, C. and Maboudian, R. (2009) 'Temperature dependence of Raman spectra for individual silicon nanowires', Phys. Rev. B, Vol. 80, No. 7, p.073306.

Doerk, G.S., Carraro, C. and Maboudian, R. (2010) 'Single nanowire thermal conductivity measurements by Raman thermography', ACS Nano, Vol. 4, No. 8, p.4908.

Hochbaum, A.I., Chen, R., Delgado, R.D., Liang, W., Garnett, E.C., Najarian, M., Majumdar, A. and Yang, P. (2008) 'Enhanced thermoelectric performance of rough silicon nanowires', Nature, Vol. 451, No. 7175, p. 163.

Kayes, B.M., Atwater, H.A. and Lewis, N.S. (2005) 'Comparison of the device physics principles of planar and radial p-n junction nanorod solar cells', J. Appl. Phys., Vol. 97, No. 11, p.114302.

Kerker, M. (1969) The Scattering of Light and other Electromagnetic Radiation, Academic Press, New York, Ch.3 and Ch.8.

Li, D., Wu, Y., Fan, R., Yang, P. and Majumdar, A. (2003a) 'Thermal conductivity of Si/SiGe superlattice nanowires', Appl. Phys. Lett., Vol. 83, No. 15, p.3186.

Li, D., Wu, Y., Kim, P., Shi, L., Yang, P. and Majumdar, A. (2003b) 'Thermal conductivity of individual silicon nanowires', Appl. Phys. Lett., Vol. 83, No. 14, p.2934.

Liu, X.F., Wang, R., Jiang, Y.P, Zhang, Q., Shan, X.Y. and Qiu, X.H. (2010) 'Thermal conductivity measurements of individual CdS nanowires using microphotoluminescence spectroscopy', J. Appl. Phys., Vol. 108, No. 5, p.054310.

Piscanec, S., Cantoro, M., Ferrari, A.C., Zapien, J.A., Lifshitz, Y., Lee, S.T., Hofmann, S. and Robertson, J. (2003) 'Raman spectroscopy of silicon nanowires', Phys. Rev. B, Vol. 68, No. 24, p.241312(R).

Soini, M., Zardo, I., Uccelli, E., Funk, S., Koblmuller, G., Fontcuberta, A. and Abstreiter, G. (2010) 'Thermal conductivity of $\mathrm{Ga}$ as nanowires studied by micro-Raman spectroscopy with laser heating', Appl. Phys. Lett., Vol. 97, No. 26, p.263107.

Torres, A., Martín-Martín, A., Martínez, O., Prieto, A.C., Hortelano, V., Jiménez, J., Rodríguez, A., Sangrador, J. and Rodríguez, T. (2010) 'Micro-Raman spectroscopy of Si nanowires: influence of diameter and temperatura', Appl. Phys. Lett, Vol. 96, No. 1, p.011904.

Xie, X.Q., Liu, W.F., Oh, J.I. and Shen, W.Z. (2011) 'Optical absorption in c-Si/a-Si:H core/shell nanowire arrays for photovoltaic applications', Appl. Phys. Lett., Vol. 99, No. 3, p.033107. 